\title{
A New Method of SMall-Signal Calibration BASED ON KALMAN FILTER
}

\author{
XU Yi-xiong, WANG Cheng-jun, XU Ya-jun, YANG Jiang-wei \\ Shanghai Aerospace Control Technology Institute, Shanghai, China
}

\begin{abstract}
The basic principle of Kalman filter $(K F)$ is introduced in this paper, based on which, it presents a new method for high precision measurement of small-signal instead of the unreal direct one. We have designed a method of multi-meter information infusion. With this method, we filter the measured value of a type of special equipment and extract the optimal estimate for true value. Experimental results show that this method can effectively eliminate the random error of the measurement process. The optimal estimate error meets the basic requirements of conformity assessment, $3 U_{95} \leq M P E V$. This method can provide an algorithm reference for the design of automatic calibration equipment.
\end{abstract}

\section{KEYWORDS}

Kalman filter; Information infusion; Multi-meter; High precision calibration

\section{INTRODUCTION}

In classical control theory, we know that the accurate measurement and feedback of signals is the premise to ensure that the system can control the relevant parameters steadily, accurately and quickly.In modern military tactical weapon measurement and control system, satellite attitude and orbit control system, the importance of high-precision signal measurement is self-evident, and its measurement method has been the focus of our research. There are two commonly used measurement methods: direct method and indirect method.Direct measurement method is often used in signal measurement with relatively low accuracy requirements. The indirect measurement principle is adopted without corresponding measuring instruments or when the accuracy of measuring instruments can not meet the requirements of use, such as physical quantity transformation measurement, comparative measurement and other methods.In this paper, a highprecision signal calibration method based on multi-instrument information fusion is proposed, which originates from the idea of federated Kalman filter.

\section{BASIC PRINCIPLES OF KALMAN FILTERING}

Essentially, Kalman filtering is a signal processing process that removes or weakens unwanted components and enhances the required components. This process can be realized either by hardware or by software. Since Kalman proposed KF in 1960 to overcome the shortcomings of Wiener filtering, it has been widely used in various fields of data processing and system control. The basic idea of Kalman filter is that the minimum mean square error is the best estimation criterion, the state space model of signal and noise is adopted, the estimation of state variables is updated by the estimation of the previous moment and the observation value of the current moment, and the estimation value of the current moment is obtained. According to the established system equation and observation equation, the algorithm makes the minimum mean square error for the signal to be processed. The process flow of signal measurement and processing is shown in Fig. 1. 


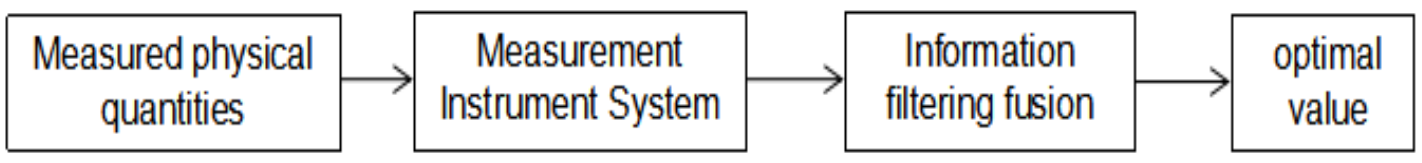

Fig. 1 Signal Measurement Processing Flow

Let the stochastic linear discrete system equation be:

$$
\begin{aligned}
& x_{k}=\Phi_{k, k-1} x_{k-1}+\Gamma_{k, k-1} w_{k-1} \\
& z_{k}=H_{k} x_{k}+v_{k}
\end{aligned}
$$

In the formula, $x_{k}$ is the n-dimension state vector of the system, ${ }^{z_{k}}$ is the m-dimensions measurement vector of the system, ${ }^{w_{k}}$ is the p-dimensions process noise of the dimension system, $v_{k}$ is the p-dimensions measurement noise, $\Phi_{k, k-1}$ is the $n \times n_{\text {dimensions state transition matrix of }}$

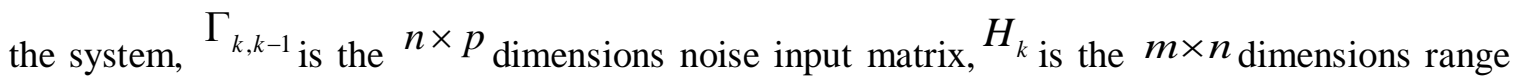
matrix and the $k$ subscript represents the $k$ moment.

Suppose that the statistics of process noise and measurement noise are as follows:

$$
\begin{gathered}
E\left(w_{k}\right)=0, E\left(w_{k} w_{j}^{T}\right)=Q_{k} \delta_{k j} \\
E\left(v_{k}\right)=0, E\left(v_{k}, v_{j}^{T}\right)=R_{k} \delta_{k j} \\
E\left(v_{k}, v_{j}^{T}\right)=S_{k}=0
\end{gathered}
$$

In the formula, $Q_{k}$ is the nonnegative definite variance matrix of system noise, ${ }^{R_{k}}$ is the positive definite variance matrix of system measurement noise and $S_{k}$ is the covariance of system noise and measurement noise.

When the estimated states and measurements satisfy (1) and (2) formulas and the system process noise and measurement noise satisfy (2), the state estimation $\hat{x}_{k}$ can be solved in the following steps:

One-step state prediction:

$$
\hat{x}_{k \mid k-1}=\Phi_{k, k-1} \hat{x}_{k-1}
$$

State estimation:

$$
\hat{x}_{k}=\hat{x}_{k \mid k-1}+K_{k}\left(z_{k}-H_{k} \hat{x}_{k \mid k-1}\right)
$$

The filter gain matrix: 


$$
K_{k}=P_{k, k-1} H_{k}^{T}\left(H_{k} P_{k \mid k-1} H_{k}^{T}+R_{k}\right)^{-1}=P_{k} H_{k}^{T} R_{k}^{T}
$$

One-step prediction error variance matrix:

$$
P_{k \mid k-1}=\Phi_{k, k-1} P_{k-1} \Phi_{k \mid k-1}^{T}+\Gamma_{k, k-1} Q_{k-1} \Gamma_{k, k-1}^{T}
$$

Estimation error variance matrix:

$$
P_{k}=\left(I-K_{k} H_{k}\right) P_{k \mid k-1}\left(I-K_{k} H_{k}\right)^{T}+K_{k} R_{k} K_{k}^{T}=\left(I-K_{k} H_{k}\right) P_{k \mid k-1}
$$

Formula (3) (7) are the basic equations of Kalman filter for stochastic linear discrete system. Given the initial value $\hat{x}_{0}$ and $\hat{p}_{0}$, the measured value ${ }^{z_{k}}$ of the $k$ time, the state estimation ${ }^{\hat{x}_{k}}$ of the $k$ time can be calculated recursively. Kalman filter estimates the state of system process by feedback control method. The filter estimates the state of the process at a certain time, and then obtains feedback by means of measurement update. One update is divided into two parts, the state update process and the measurement update process.

\section{INFORMATION FUSION METHOD OF MULTI-MEASURING INSTRUMENTS}

When it is necessary to measure high-precision signals, according to Kalman's basic principle, multi-instrument information fusion can be used to improve the accuracy of measurement results.In this paper, the 8-bit and-a-half high-precision digital meter F3458A (8-bit and a half) is used as the main measuring instrument, and several sub-measuring instruments can be added for filtering, such as F8846A, Keithley 2001 (six and a half), Keithley 2000 (six and a half), Agilent 34405A (five and a half), F8808A (five and a half)...The $n-1$ sub-filter is fused with the main measuring instrument 3458A separately, and then the n-1 sub-information and the main information are fused with the main filter again to get the optimal value of the measured value. The algorithm structure is shown in Figure 2.

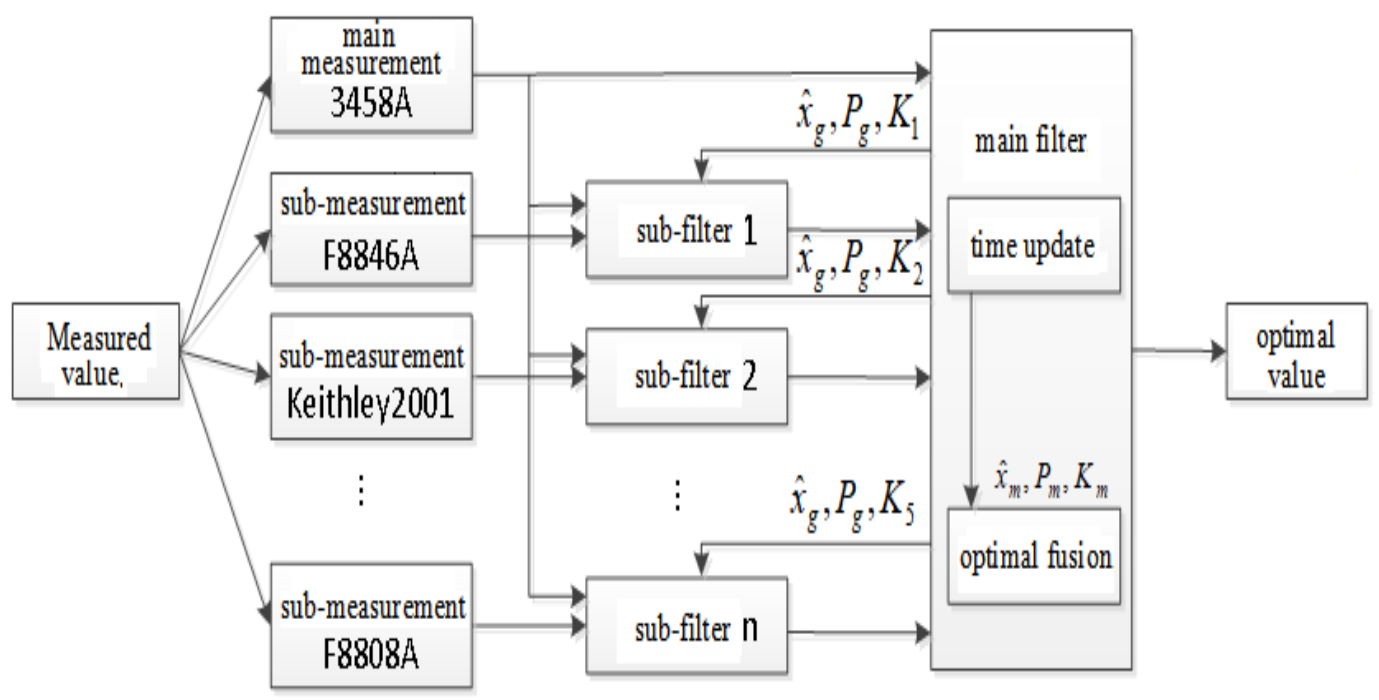

Fig. 2 Algorithmic structure of multi-digit table information fusion filtering 


\subsection{ONE-DIMENSIONAL FILTER DESIGN}

Assuming that the theoretical value of measurement is $25 \mathrm{uV}$, the error requirement is $1 \mathrm{uV}$, and the error of measurement instrument is $1.5 \mathrm{uV}$, a single instrument is used to measure the voltage of $25 \mathrm{uV} 60$ times in a short time. The measured value contains random noise which obeys Gauss distribution. By Kalman filter, the measurement results and measurement errors are compared, as shown in Figure 3.

The state equation of one-dimensional filter is:

$$
\begin{aligned}
& x_{k}=x_{k-1}+w_{k-1} \\
& z_{k}=x_{k}+v_{k}
\end{aligned}
$$

In the formula, the state matrix $=1$, the noise input matrix $=1$, the covariance $=0.01$, and the measurement matrix $=1$.
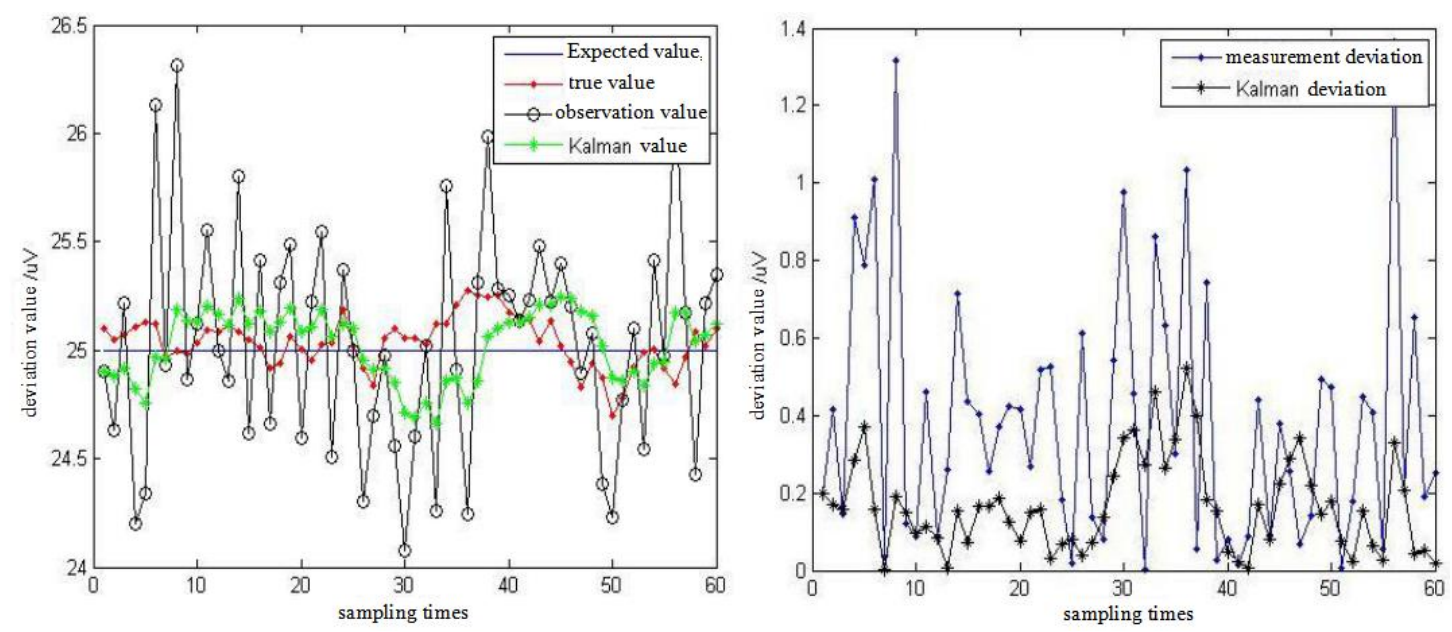

Fig. 3 Comparison of measurement results and errors of one-dimensional filtering

As can be seen from Figure 3, in practice, there is always a random variation of the real value around the expected value when the signal source outputs the expected value; the observed curve has poor tracking ability to the real value, and it has strong tracking ability after Kalman filtering. From the right figure, it can be seen more intuitively that the measurement deviation of the observation value is 1.247 , the measurement deviation after Kalman filtering is 0.526, and the tracking ability before and after Kalman filtering is better than that before Kalman filtering. The measurement accuracy is increased by more than one time.

\subsection{SIX-DIMENSIONAL FILTER DESIGN}

Fig. 2 is used to construct a six-dimensional filter to fuse the information of six measuring instruments. According to the measurement accuracy and resolution of each measuring instrument, the elements of state matrix and measurement matrix, i.e. weights, are set, and the elements of noise input matrix and covariance are set according to the measurement error.

The six-bit filter equation of state is: 


$$
\begin{aligned}
& x_{k}=\Phi_{k, k-1} x_{k-1}+\Gamma_{k, k-1} w_{k-1} \\
& z_{k}=H_{k} x_{k}+v_{k}
\end{aligned}
$$

The state matrix, noise input matrix and measurement matrix are all $6 \times 6$-dimensional diagonal matrices, and they are all $6 \times 1$-dimensional noise input. The covariance is $6 \times 1$-dimensional. The simulation results are shown in Fig. 4.
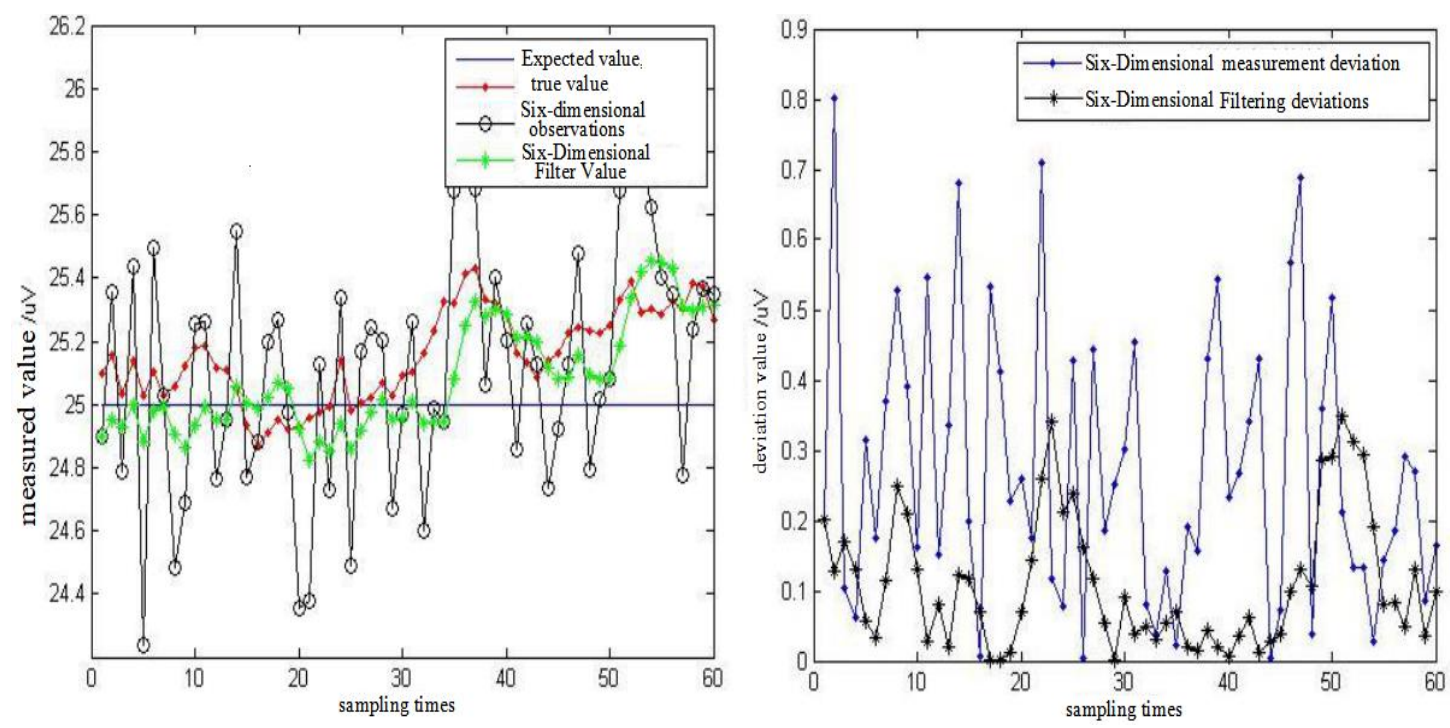

Fig. 4 Six-Dimensional Filtering Measurement Results

From Figure 4, we can see that the measurement accuracy of six-dimensional observations and Kalman filtering have been improved, and the tracking ability of real values has been strengthened. The measurement deviation after fusion of six-dimensional observations is 0.815 , and that after Kalman filtering is 0.331 . At this time, the error of the best estimation has met the basic requirements of conformity evaluation, that is, the error of the best estimation has met the basic requirements of conformity evaluation. The measurement accuracy of six-dimensional Kalman filter is about four times higher than that of one-dimensional observation.

In summary, under this measurement system model, increasing the number of information fusion instruments can effectively improve the measurement accuracy of observation value and Kalman filter. After the follow-up experiments and simulations, according to the types of conventional measuring instruments, the measurement accuracy of Kalman filter can be improved by one accuracy grade compared with that of the main measuring instrument when it is increased to 8 fusion instruments.

\section{SUMMARY}

In this paper, based on the basic idea of Kalman filter (KF), an extended Kalman filter (EKF) algorithm is designed by using multi-source information fusion technology, and a new method of small signal high precision measurement is proposed. However, this method has a large amount of field data acquisition and is inconvenient for manual calculation. Therefore, this algorithm provides a reference for the design of automatic measurement system. 


\section{REFERENCES}

[1] Pan Quan, Cheng Yongmei, Liang Yan, et al. Theory and application of multi-source information fusion [M]. Beijing: Tsinghua University Press, 2013

[2] He You, Wang Guohong, et al. Multisensor Information Fusion and Application (Second Edition) [M]. Beijing: Electronic Industry Press, 2007.

[3] China Metrology and Testing Association. Professional Practice of Measurement Data Processing and Measurement [M]. Beijing: China Quality Inspection Press, 2013.

[4] Yixiong Xu. The Pressure Signal Calibration Technology of the Comprehensive Test System[J]. Informatics Engineering, an International Journal, Vol.4, N0.2,June 2016:01 08.

[5] Chen Kui. Experimental Design and Analysis [M]. Beijing: Tsinghua University Press (2nd edition), 2005

[6] Li Jing, Xu Hai. Platform recognition algorithm based on heterogeneous sensor information fusion [J]. Ship ECM, 2016 [6]: 33-42.

[7] Chen H M, Kirubarjan T, Bar-Shalom T Track-to-track fusion versus centralized estimation: Theory and application. IEEE Transactions on Aerospace and Electronic System, 2008, 39(2):386-411.

\section{AUTHORS}

Xu Yi-xiong(1985-), Shanghai Aerospace Control Technology Institute, the main research interest now is Instrumentation measurement technology, address: Room 1105,Building NO.243,Lane 777,Dushi road, Minhang district, Shanghai(201109), fixed 021-34628108.

WANG Cheng-jun(1983-), Shanghai Aerospace Control Technology Institute, the main research interest now is Inspection technology, address: Room 405,Building NO.2,Lane 1555,Zhong Chun road, Minhang district, Shanghai(201109), fixed 02124184425.

XU Ya-jun(1987-), Shanghai Aerospace Control Technology Institute, the main research interest now is alignment technology, address: Room 405,Building NO.2,Lane 1555,Zhong Chun road, Minhang district, Shanghai(201109), fixed 021-24184409.

Yang Jiang-wei(1983-), Shanghai Aerospace Control Technology Institute, the main research interest now is Engineering Technology, address: Room 405,Building NO.2,Lane 1555,Zhong Chun road, Minhang district, Shanghai(201109), fixed 02124184207.
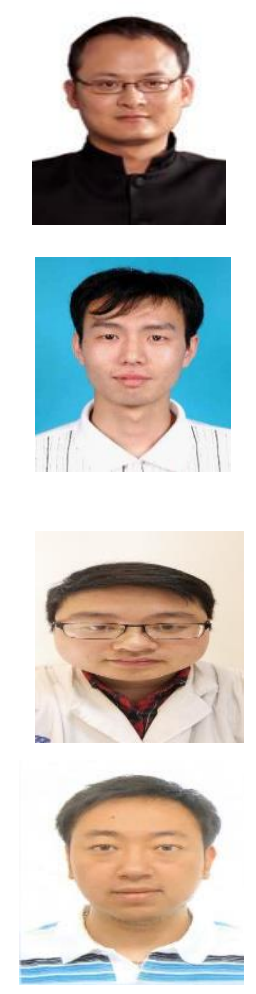